were characterized by facial grimacing, head twitching, and shoulder shrugging plus eye rolling, facial contorsions, jumping, touching objects and body parts and making obscene gestures. Vocal tics included grunting, sniffing, and snorting sounds. The tics were suppressed after several weeks on Pimozide (Orap $1 \mathrm{mg}$ b.i.d.). (Northam RS, Singer HS. Postencephalitic acquired Tourette-like syndrome in a child. Neurology April 1991; $41: 592-593$ ).

COMNENT. The authors refer to reports of acquired Tourette syndrome in adults. Tics followed withdrawal from neuroleptic medications or in association with herpes and other encephalitides, toxic and metabolic encephalopathies, strokes, cerebral tumors, trauma, multiple sclerosis, syphilis, Huntington's, Alzheimer's, and Creutzfeldt-Jakob diseases.

\title{
SPINAL CORD SCHISTOSOMIASIS
}

A seven year old girl with Schistosoma mansoni infection of the spinal cord mimicking cord neoplasm is reported from the Department of Neurology, University of Michigan, Ann Arbor, MI. Exposure to Schistosomal parasites occurred while bathing in a river in Sierra Leone, Southern Africa. The illness presented with back and leg pain and a low-grade fever. Within 24 hours she had weakness of the leg and four days later she was unable to walk, to urinate or defecate. Sensation was diminished below the low thoracic area. CSF showed 2 RBCs, 250 WBCs with $78 \%$ lymphocytes, protein $584 \mathrm{mg} / \mathrm{dl}$ and glucose $55 \mathrm{mg} / \mathrm{dl}$. Myelography revealed a large intramedullary mass at thoracic levels 11 and 12. MRI with Gadolinium demonstrated enhancement of the cord at T10 level through the conus. Decompressive laminectamy was performed with resection of a yellow gliotic appearing cord of neoplastic appearance. Histologic examination revealed eggs characteristic of $S$. mansoni. After treatment with praziquantel, lower extremity strength gradually improved and six months later, she was walking well with braces. (Selwa LM, Garofalo EA et al. Spinal cord schistosomiasis: A pediatric case mimicking intrinsic cord neoplasm. Neurology May 1991; 41:755-757).

COMMENT. In patients with acute or subacute paraparesis who have been exposed to endemic areas (South Africa, South America, Caribbean or the Middle East), schistosomiasis must be suspected. Spinal cord involvement can be delayed for up to six years after exposure, and findings on MRI and myelography may mimic spinal cord neoplasms. Patients with mild or early involvement of the spinal cord may be benefitted by antiparasitic medications alone and surgery may be unnecessary.

\section{CNS COMPLICATIONS OF RHEUMATOID ARTHRITIS}

Mass lesions in the basal ganglia bilaterally and mainly involving the globus pallidus are reported in a two year old boy with juvenile rheumatoid arthritis from the Department of Pediatrics, Hokushin General Hospital, Nishi, Nagano, Japan. At ten months after the onset of the arthritis he developed a relapse with fever, heart murmur, 